Volume 359, Number 12, December 2007, Pages 5915-5929

S 0002-9947(07)04178-5

Article electronically published on July 23, 2007

\title{
THE DIVERGENCE THEOREM FOR UNBOUNDED VECTOR FIELDS
}

\author{
THIERRY DE PAUW AND WASHEK F. PFEFFER
}

\begin{abstract}
In the context of Lebesgue integration, we derive the divergence theorem for unbounded vector fields that can have singularities at every point of a compact set whose Minkowski content of codimension greater than two is finite. The resulting integration by parts theorem is applied to removable sets of holomorphic and harmonic functions.
\end{abstract}

In the context of Lebesgue integration, powerful divergence theorems (alternatively called Gauss-Green theorems) have been proved in $[5,6,11]$ for bounded vector fields. Using generalized Riemann integrals, divergence theorems for certain kinds of unbounded vector fields are established in [12] and [10]. These vector fields are allowed to exhibit a controlled growth to infinity in neighborhoods of points lying on small sets - planes of codimension greater than two in [12], and compact sets of finite Hausdorff measure of codimension greater than two in [10]. However, a closer look at these results shows that, due to the growth conditions imposed, they apply only to vector fields with a finite number of singular points (see Remark 2.4 below for details).

In contrast, we obtain a divergence theorem for vector fields having singularities at all points of certain compact sets of finite upper Minkowski content of codimension greater than two (see the Main Theorem below). This is achieved by imposing growth conditions about exceptional sets, rather than about individual points of exceptional sets. We employ only the Lebesgue integral, and integrate over bounded BV sets. Our result generalizes substantially the divergence theorem of [11]. As in [11], we show here that mere integrability of divergence implies the Gauss-Green formula; the separate integrability of the relevant partial derivatives is not required. In view of this, we can employ vector fields whose coordinates are not BV functions (see the paragraph following Example 2.6 below).

Whether Minkowski contents can be replaced by Hausdorff measures is unclear. The techniques used in [7, Section 3] for characterizing removable sets of linear partial differential equations in terms of Hausdorff measures yield results different from ours (cf. Theorems 4.2 and 4.3 below), and they do not appear directly applicable in our approach.

Received by the editors August 11, 2005.

2000 Mathematics Subject Classification. Primary 26B20; Secondary 26B05, 28A75.

Key words and phrases. BV sets, Hausdorff measures, Minkowski contents.

The first author was a chercheur qualifié of the Fonds National de la Recherche Scientifique in Belgium.

The second author was supported in part by the Université Catholique de Louvain in Belgium. 
The paper is organized as follows. Section 1 contains necessary preliminaries, such as the definitions of BV sets, the upper Minkowski content, and the resulting dimension. It also includes the definition of transversality between a compact set and the essential boundary of a BV set. In Section 2, we introduce derivatives of vector fields defined on measurable sets and formulate the divergence theorem, which is proved in Section 3. The integration by parts formula is derived in Section 4, and it is applied to removable sets of the Cauchy-Riemann and Laplace equations.

\section{Preliminaries}

The set of all real numbers is denoted by $\mathbb{R}$, and $\mathbb{R}_{+}:=\{t \in \mathbb{R}: t>0\}$. Unless specified otherwise, by a function we always mean a real-valued function. Countable sets are finite (including the empty set) or countably infinite.

The ambient space of this note is $\mathbb{R}^{m}$ where $m \geq 1$ is a fixed integer. The usual inner product $x \cdot y$ induces a norm $|\cdot|$ in $\mathbb{R}^{m}$, and the boldface zero $(\mathbf{0})$ denotes the zero vector of $\mathbb{R}^{m}$. A cube is a compact nondegenerate cube in $\mathbb{R}^{m}$.

The closure, interior, and diameter of a set $E \subset \mathbb{R}^{m}$ are denoted by $\mathrm{cl} E$, int $E$, and $d(E)$, respectively. The distance from a point $x \in \mathbb{R}^{m}$ to a set $E \subset \mathbb{R}^{m}$ is denoted by $d(x, E)$. For a set $E \subset \mathbb{R}^{m}$ and $r>0$, we let

$$
B(E, r):=\left\{x \in \mathbb{R}^{m}: d(x, E)<r\right\}
$$

and write $B(z, r)$ instead of $B(\{z\}, r)$ if $E=\{z\}$.

In $\mathbb{R}^{m}$ we use Lebesgue measure $\mathcal{L}^{m}$, and the Hausdorff measures $\mathcal{H}^{t}$ where $0 \leq t \leq m$ is a real number [3, Section 2.1]. For $E \subset \mathbb{R}^{m}$, we write $|E|$ instead of $\mathcal{L}^{m}(E)=\mathcal{H}^{m}(E)$. The restricted measures $\mathcal{L}^{m} L E$ and $\mathcal{H}^{t} L E$ are defined in the usual way [3, Section 1.1.1]. Unless specified otherwise, the words "measure," "measurable," and "negligible," as well as the expressions "almost everywhere" and "almost all" refer to Lebesgue measure $\mathcal{L}^{m}$.

Let $E \subset \mathbb{R}^{m}$, and for $0 \leq \theta \leq 1$, let

$$
E(\theta):=\left\{x \in \mathbb{R}^{m}: \lim _{r \rightarrow 0+} \frac{|B(x, r) \cap E|}{|B(x, r)|}=\theta\right\} .
$$

The sets $\operatorname{int}_{*} E:=E(1), \mathrm{cl}_{*} E:=\mathbb{R}^{m}-E(0)$, and $\partial_{*} E:=\mathrm{cl}_{*} E-\mathrm{int}_{*} E$ are called, respectively, the essential interior, essential closure, and essential boundary of $E$. The relevant closure of $E$ is the set $\mathrm{cl}_{r} E:=\mathrm{cl}\left(\mathrm{cl}_{*} E\right)$. The extended real number $\|E\|:=\mathcal{H}^{m-1}\left(\partial_{*} E\right)$ is called the perimeter of $E$.

A set of finite perimeter, or a $B V$ set, is a measurable set $A \subset \mathbb{R}^{m}$ with $|A|<\infty$ and $\|A\|<\infty$. Although some of our partial results hold for any BV set, in what follows we deal exclusively with the family $\mathcal{B} \mathcal{V}$ of all bounded BV sets. If $A \in \mathcal{B} \mathcal{V}$, then $\mathcal{H}^{m-1}$ almost everywhere in $\partial_{*} A$ is defined a unit vector field $\nu_{A}$ such that

$$
\int_{A} \operatorname{div} v d \mathcal{L}^{m}=\int_{\partial_{*} A} v \cdot \nu_{A} d \mathcal{H}^{m-1}
$$

for each $v \in C^{1}\left(\mathbb{R}^{m} ; \mathbb{R}^{m}\right)$; the vector field $\nu_{A}$, called the unit exterior normal of $A$, is unique up to an $\mathcal{H}^{m-1}$ negligible subset of $\partial_{*} A$.

Our goal is to relax the boundedness condition in the following theorem, whose elementary proof can be found in [11]. 
Theorem 1.1 (Gauss-Green). Let $A \in \mathcal{B} \mathcal{V}$, and let $v: \mathrm{cl}_{*} A \rightarrow \mathbb{R}^{m}$ be bounded. Assume that $E_{c}$ and $E_{d}$ are subsets of $\mathrm{cl}_{*} A$ such that

(1) $E_{c}$ is $\mathcal{H}^{m-1}$ negligible, and $E_{d}$ is $\mathcal{H}^{m-1} \sigma$-finite,

(2) $|v(y)-v(x)|=o(1)$ as y approaches $x \in \mathrm{cl}_{*} A-E_{c}$,

(3) $|v(y)-v(x)|=O(1)|y-x|$ as $y$ approaches $x \in \mathrm{cl}_{*} A-E_{d}$.

Then $v \in L^{1}\left(\partial_{*} A, \mathbb{R}^{m} ; \mathcal{H}^{m-1}\right)$, and there is a measurable function div $v$ defined almost everywhere in $A$ that is uniquely determined by $v$ up to a negligible set. Moreover, if $\operatorname{div} v \in L^{1}\left(A ; \mathcal{L}^{m}\right)$, then

$$
\int_{A} \operatorname{div} v d \mathcal{L}^{m}=\int_{\partial_{*} A} v \cdot \nu_{A} d \mathcal{H}^{m-1} .
$$

The function $\operatorname{div} v$ in Theorem 1.1 is defined either by means of Whitney's and Stepanoff's theorems as in [11, Proof of Theorem 2.9], or using relative differentiation introduced in Section 2 below (Definition 2.1 and Proposition 2.3). It is easy to verify that both definitions of $\operatorname{div} v$ coincide almost everywhere in $A$. We note that under the assumptions of Theorem 1.1, the classical divergence of $v:=\left(v_{1}, \ldots, v_{m}\right)$ equal to $\sum_{i=1}^{m} \partial v_{i} / \partial x_{i}$ may not be defined at any $x \in A$. However, if $x$ is an interior point of $A$ and $v$ is differentiable at $x$, then $\operatorname{div} v(x)$ of Theorem 1.1 is defined and equals the classical divergence of $v$ at $x$.

For $0 \leq t \leq m$, the $t$-dimensional upper Minkowski content of a set $E \subset \mathbb{R}^{m}$ is the extended real number

$$
\mathcal{M}^{* t}(E):=\limsup _{r \rightarrow 0+} \frac{|B(E, r)|}{r^{m-t}} .
$$

Clearly, $\mathcal{M}^{* 0}(E)=0$ if and only if $E$ is empty, and $\mathcal{M}^{* 0}(E)<\infty$ if and only if $E$ is finite. There is a $\kappa>0$, depending only on $m$ and $t$, such that $\mathcal{H}^{t}(E) \leq \kappa \mathcal{M}^{* t}(E)$ for each $E \subset \mathbb{R}^{m}\left[9\right.$, Section 5.5]. As $\mathcal{M}^{* t}(E)=\mathcal{M}^{* t}(\operatorname{cl} E)$ and $\mathcal{M}^{* t}(E)=\infty$ whenever $E$ is unbounded, we apply upper Minkowski contents only to compact sets.

Let $K \subset \mathbb{R}^{m}$ be a compact set, and let $0 \leq t \leq m$. The following facts are self-evident:

(i) if $\mathcal{M}^{* t}(K)>0$, then $\mathcal{M}^{* s}(K)=\infty$ whenever $0 \leq s<t$;

(ii) if $\mathcal{M}^{* t}(K)<\infty$, then $\mathcal{M}^{* s}(K)=0$ whenever $t<s \leq m$.

For $K \neq \emptyset$, it follows that

$$
\sup \left\{s \in[0, m]: \mathcal{M}^{* s}(K)>0\right\}=\inf \left\{s \in[0, m]: \mathcal{M}^{* s}(K)<\infty\right\},
$$

and we denote this common value by $\operatorname{dim} K$; for $K=\emptyset$, we let $\operatorname{dim} K:=-\infty$. Clearly $\operatorname{dim} K=t$ whenever $0<\mathcal{M}^{* t}(K)<\infty$. It is easy to see that $\operatorname{dim} K=0$ whenever $K$ is nonempty and finite, but the converse is false.

Remark 1.2. To identify the value of $\mathcal{M}^{* t}(K)$ with that of $\mathcal{H}^{t}(K)$ for a nice set $K$, a positive multiplicative constant depending on $m$ and $t$ is usually included in the definition of $\mathcal{M}^{* t}(K)$. Since we are interested only in distinguishing the cases $\mathcal{M}^{* t}(K)=0,0<\mathcal{M}^{* t}(K)<\infty$, and $\mathcal{M}^{* t}(K)=\infty$, no such constant is necessary for our purposes.

Example 1.3. Let $C$ be the Cantor ternary set placed on any line $\ell$ in $\mathbb{R}^{m}$, and let $t=\log 2 / \log 3$. Since $\mathcal{H}^{t}(C)>0$ [4, Theorem 1.14], we have $\mathcal{M}^{* t}(C)>0$. To show $\mathcal{M}^{* t}(C)<\infty$, recall that $C=\bigcap_{k=1}^{\infty} C_{k}$ where each $C_{k}$ is the union of $2^{k}$ disjoint 
compact segments of $\ell$ such that the length of each is $3^{-k}$ and the distance between any two is greater than or equal to $3^{-k}$. Choose a positive $r<1 / 6$, and find a positive integer $k$ so that $3^{-k-1} \leq 2 r<3^{-k}$. It is easy to see that

$$
\left|B\left(C_{k}, r\right)\right|<\kappa 2^{k} 3^{-m k}
$$

where $\kappa$ is a constant depending only on the dimension $m$. Moreover $2 / 3^{t}=1$ and $r^{m-t} \geq 3^{-(k+1)(m-t)} / 2^{m-t}$, which implies

$$
\frac{|B(C, r)|}{r^{m-t}} \leq \frac{\left|B\left(C_{k}, r\right)\right|}{r^{m-t}}<\kappa 6^{m} .
$$

Thus $\mathcal{M}^{* t}(C) \leq \kappa 6^{m}<\infty$, and we conclude $\operatorname{dim} C=t$.

Definition 1.4. Let $A \in \mathcal{B V}$, let $K \subset \mathbb{R}^{m}$ be a nonempty compact set, and let $t:=\operatorname{dim} K$. We say that $K$ is transverse to $\partial_{*} A$ if there is a decreasing function $\beta: \mathbb{R}_{+} \rightarrow \mathbb{R}_{+}$such that $\int_{0}^{1} \beta(r) d r<\infty$ and

$$
\limsup _{r \rightarrow 0+} \frac{\mathcal{H}^{m-1}\left[B(K, r) \cap \partial_{*} A\right]}{\beta(r) r^{m-t}}<\infty .
$$

Let $A \in \mathcal{B} \mathcal{V}$, and let $K \subset \mathbb{R}^{m}$ be a nonempty compact set. If $K \cap \operatorname{cl}\left(\partial_{*} A\right)=\emptyset$, then clearly $K$ is transverse to $\partial_{*} A$. Letting $\beta(r):=r^{t-m}$, we see that $K$ is transverse to $\partial_{*} A$ whenever $\operatorname{dim} K>m-1$. On the other hand, [3, Section 2.3, Theorem 2] and Observation 1.6 below show that for $\mathcal{H}^{m-1}$ almost all $x \in \partial_{*} A$, the singleton $\{x\}$ is not transverse to $\partial_{*} A$.

Observation 1.5. Let $A \in \mathcal{B} \mathcal{V}$, and let $K \subset \mathbb{R}^{m}$ be a nonempty compact set transverse to $\partial_{*} A$. If $\mathcal{M}^{* s}(K)<\infty$, then there is a decreasing function $\beta: \mathbb{R}_{+} \rightarrow \mathbb{R}_{+}$ such that $\int_{0}^{1} \beta(r) d r<\infty$ and

$$
\limsup _{r \rightarrow 0+} \frac{\mathcal{H}^{m-1}\left[B(K, r) \cap \partial_{*} A\right]}{\beta(r) r^{m-s}}<\infty .
$$

Proof. Since $t:=\operatorname{dim} K \leq s$, taking $\beta$ as in Definition 1.4 yields

$$
\limsup _{r \rightarrow 0+} \frac{\mathcal{H}^{m-1}\left[B(K, r) \cap \partial_{*} A\right]}{\beta(r) r^{m-s}}=\limsup _{r \rightarrow 0+} \frac{\mathcal{H}^{m-1}\left[B(K, r) \cap \partial_{*} A\right]}{\beta(r) r^{m-t}} \cdot r^{s-t}<\infty .
$$

Observation 1.6. If $\beta: \mathbb{R}_{+} \rightarrow \mathbb{R}_{+}$is a decreasing function and $\int_{0}^{1} \beta(r) d r$ is finite, then $\lim _{r \rightarrow 0+} r \beta(r)=0$.

Proof. Proceeding toward a contradiction, suppose $r_{k} \beta\left(r_{k}\right) \geq c>0$ for a strictly decreasing sequence $\left\{r_{k}\right\}$ in $(0,1)$ with $\lim r_{k}=0$, and let $s_{k}:=r_{k+1} / r_{k}$. Since

$$
\int_{r_{k+1}}^{r_{k}} \beta(r) d r \geq \beta\left(r_{k}\right)\left(r_{k}-r_{k+1}\right) \geq c\left(1-s_{k}\right),
$$

the series $\sum_{k=1}^{\infty}\left(1-s_{k}\right)$ converges. By [14, Theorem 15.5],

$$
\lim _{k \rightarrow \infty} \frac{r_{k}}{r_{1}}=\lim _{k \rightarrow \infty} \prod_{i=1}^{k-1} s_{i}=\prod_{k=1}^{\infty} s_{k}>0
$$

contrary to our assumption. 
Remark 1.7. Assume $\partial_{*} A$ and $K$ are $C^{\infty}$ manifolds and $\operatorname{dim} K \leq m-1$. If $K$ is transverse to $\partial_{*} A$ in the sense of differential topology [8, Chapter 3], then $K$ is also transverse to $\partial_{*} A$ according to Definition 1.4. However, in the measure theoretic setting, the geometric content of transversality is not obvious. Distinct definitions may be introduced, which agree on $C^{\infty}$ manifolds. For instance, in view of Observation 1.6, inequality (1.2) may be relaxed to

$$
\lim _{r \rightarrow 0+} \frac{\mathcal{H}^{m-1}\left[B(K, r) \cap \partial_{*} A\right]}{r^{m-1-t}}=0 .
$$

The definition we have chosen leads to an integration by parts theorem (Theorem 4.1 below) suitable for our applications.

The next definition introduces a concept related to transversality, albeit only formally. The reader should compare it with Example 1.9, Remark 2.7, and Example 2.8 below.

Definition 1.8. Let $A \in \mathcal{B} \mathcal{V}$, let $K \subset \mathbb{R}^{m}$ be a nonempty compact set, and let $t:=\operatorname{dim} K$. We say that $\partial_{*} A$ accumulates about $K$ if

$$
\limsup _{r \rightarrow 0+} \frac{\mathcal{H}^{m-1}\left[B(K, r) \cap \partial_{*} A\right]}{r^{m-1-t}}=\infty .
$$

Let $A \in \mathcal{B} \mathcal{V}$. The set of all $x \in \mathbb{R}^{m}$ such that $\partial_{*} A$ accumulates about $\{x\}$ is called the singular boundary of $A$, denoted by $\partial_{s} A$. It follows from [3, Section 2.3] that $\mathcal{H}^{m-1}\left(\partial_{s} A\right)=0$.

Example 1.9. Let $m=2$, and let

$$
A_{k}:=\left\{x \in \mathbb{R}^{2}:\left(k+\varepsilon_{k}\right)^{-2} \leq|x| \leq k^{-2}\right\}
$$

where $0<\varepsilon_{k}<1$. The set $A:=\bigcup_{k=1}^{\infty} A_{k}$ is BV, and $\mathbf{0}$ belongs to $\operatorname{int}_{*} A$ or $\mathbb{R}^{2}-\mathrm{cl}_{*} A$ according to whether $\lim \varepsilon_{k}$ equals 1 or 0 , respectively. In either case, $\partial_{s} A=\{\mathbf{0}\}$.

\section{THE RESULT}

As BV sets of positive measure may have no interior points, we need to extend the definition of derivative to points of essential interior.

Definition 2.1. Let $E \subset \mathbb{R}^{m}, x \in$ int $_{*} E$, and $v: \mathrm{cl}_{*} E \rightarrow \mathbb{R}^{m}$. We say that $v$ is relatively differentiable at $x$ if there is a linear map $D v(x): \mathbb{R}^{m} \rightarrow \mathbb{R}^{m}$ such that

$$
|v(y)-v(x)-D v(x)(y-x)|=o(1)|y-x|
$$

as $y \in \mathrm{cl}_{*} E$ approaches $x$.

Relatively differentiable maps are approximately differentiable in the sense of $[5$, Section 3.1.2]. Although the converse is false, relative and approximate differentiability exhibit many similarities. In particular, an argument analogous to proving the uniqueness of approximate derivatives [3, Section 6.1.3, Theorem 3] shows that the linear map $D v(x)$ of Definition 2.1 is unique; see [13, Observation 2.5.6] for details. The number

$$
\operatorname{div} v(x):=\text { trace of } D v(x)
$$

is called the relative divergence of $v$ at $x$. Since our concept of relative differentiability coincides with the usual definition of differentiability whenever $x$ is the interior point of $E$, using the standard notation will cause no confusion. 
Let $B \subset E \subset \mathbb{R}^{m}$ and $x \in \operatorname{int}_{*} B$. If $v: \mathrm{cl}_{*} E \rightarrow \mathbb{R}^{m}$ is relatively differentiable at $x$, then so is the restriction $w:=v\left\lceil\mathrm{cl}_{*} B\right.$ and $D w(x)=D v(x)$. In other words, relative differentiation commutes with restrictions:

$$
D\left(v \uparrow \mathrm{cl}_{*} B\right)=(D v) \uparrow \operatorname{int}_{*} B .
$$

Applying arguments similar to those used for approximate differentiation in $[5$, Section 3.3], we obtain a version of Stepanoff's theorem for relative differentiability (Proposition 2.3 below).

Lemma 2.2. Let $E \subset \mathbb{R}^{m}$ and $C \subset \operatorname{int}_{*} E$. Suppose $v: \mathrm{cl}_{*} E \rightarrow \mathbb{R}^{m}$ satisfies the following conditions:

(i) there are positive numbers $c$ and $\delta$ such that

$$
|v(x)-v(y)| \leq c|x-y|
$$

for each $y \in C$ and each $x \in B(y, \delta) \cap \mathrm{cl}_{*} E$;

(ii) the restriction $v \uparrow C$ is Lipschitz.

If a Lipschitz extension $w: \mathbb{R}^{m} \rightarrow \mathbb{R}^{m}$ of $v \uparrow C$ is differentiable at $z \in C \cap \operatorname{int}_{*} C$, then $v$ is relatively differentiable at $z$ and $D v(z)=D w(z)$.

Proof. Select a Lipschitz extension $w: \mathbb{R}^{m} \rightarrow \mathbb{R}^{m}$ of $v \uparrow C$ and a $z \in C \cap \operatorname{int}_{*} C$ at which $w$ is differentiable. Choose a positive $\varepsilon \leq 1$, and making $c$ larger and $\delta$ smaller, assume $\operatorname{Lip}(w) \leq c, \delta<\varepsilon$, and

$$
|w(x)-w(z)-D w(z)(x-z)| \leq \varepsilon|x-z|
$$

for each $x \in B(z, \delta)$. Let $r_{x}:=|x-z|$, and observe

$$
\lim _{x \rightarrow z} \frac{\left|B\left(x, \varepsilon r_{x}\right) \cap C\right|}{\left|B\left(x, \varepsilon r_{x}\right)\right|}=1
$$

[13, Lemma 1.5.6]. Thus making $\delta$ still smaller, we may assume $B\left(x, \varepsilon r_{x}\right) \cap C \neq \emptyset$ for each $x \in B(z, \delta)$. Given $x \in B(z, \delta) \cap \mathrm{cl}_{*} E$ with $x \neq z$, choose a $y \in B\left(x, \varepsilon r_{x}\right) \cap C$. Since $w(z)=v(z), w(y)=v(y)$, and $|y-x|<\varepsilon|x-z|<\delta<\varepsilon$, we obtain

$$
\begin{aligned}
& |v(x)-v(z)-D w(z)(x-z)| \\
& \quad \leq|v(x)-v(z)-[w(x)-w(z)]|+|w(x)-w(z)-D w(z)(x-z)| \\
& \quad \leq|v(x)-v(y)|+|w(y)-w(x)|+\varepsilon|x-z| \leq \varepsilon(2 c+1)|x-z| .
\end{aligned}
$$

Proposition 2.3. Let $E \subset \mathbb{R}^{m}, C \subset \operatorname{int}_{*} E$, and $v: \mathrm{cl}_{*} E \rightarrow \mathbb{R}^{m}$. If for each $x \in C$,

$$
|v(y)-v(x)|=O(1)|y-x|
$$

as $y \in \mathrm{cl}_{*} E$ approaches $x$, then $v$ is relatively differentiable at almost all $x \in C$.

Proof. For $i=1,2, \ldots$, denote by $C_{i}$ the set of all $x \in C$ such that

$$
|v(x)-v(y)| \leq i|x-y|
$$

for each $y \in B(x, 1 / i) \cap \mathrm{cl}_{*} E$, and observe $C=\bigcup_{i=1}^{\infty} C_{i}$. Each set $C_{i}$ is the union of sets $C_{i, j}$ with $d\left(C_{i, j}\right)<1 / i$ for $j=1,2, \ldots$ It follows that each $v \uparrow C_{i, j}$ is Lipschitz. Indeed given $x, y \in C_{i, j}$, we have

$$
|v(x)-v(y)| \leq i|x-y|
$$


since $x \in C_{i}$ and $y \in B(x, 1 / i) \cap \mathrm{cl}_{*} E$. By Rademacher's theorem [3, Section 3.1.2, Theorem 2], a Lipschitz extension $w_{i, j}$ of $v \uparrow C_{i, j}$ to $\mathbb{R}^{m}$ is differentiable almost everywhere. Lemma 2.2 implies that $v$ is relatively differentiable at almost all $x \in C_{i, j}$, and hence at almost all $x \in C$.

Main Theorem (Gauss-Green). Let $A \in \mathcal{B} \mathcal{V}$ and $v: \mathrm{cl}_{*} A \rightarrow \mathbb{R}^{m}$. Assume that $E_{c}$ and $E_{d}$ are subsets of $\mathrm{cl}_{*} A$ such that

(1) $E_{c}$ is $\mathcal{H}^{m-1}$ negligible, and $E_{d}$ is $\mathcal{H}^{m-1} \sigma$-finite,

(2) $|v(y)-v(x)|=o(1)$ as $y$ approaches $x \in \mathrm{cl}_{*} A-E_{c}$,

(3) $|v(y)-v(x)|=O(1)|y-x|$ as $y$ approaches $x \in \mathrm{cl}_{*} A-E_{d}$.

In addition, assume that there is a countable collection $\left\{K_{i}\right\}$ of disjoint compact subsets of $E_{c} \cup\left(\mathrm{cl}_{r} A-\mathrm{cl}_{*} A\right)$ satisfying the following conditions:

(4) each $K_{i}$ is nonempty and transverse to $\partial_{*} A$;

(5) for each $K_{i}$, there is a nonnegative $t_{i}<m-1$ with $\mathcal{M}^{* t_{i}}\left(K_{i}\right)<\infty$;

(6) $v$ is bounded in $\mathrm{cl}_{*} A-V$ for each open set $V$ containing $\bigcup_{i} K_{i}$;

(7) for each $K_{i}$, as y approaches $K_{i}$,

$$
|v(y)|=o(1) d\left(y, K_{i}\right)^{t_{i}+1-m} \quad \text { or } \quad|v(y)|=O(1) d\left(y, K_{i}\right)^{t_{i}+1-m}
$$

according to whether $\mathcal{M}^{* t_{i}}\left(K_{i}\right)>0$ or $\mathcal{M}^{* t_{i}}\left(K_{i}\right)=0$, respectively.

Then $v$ and $v \uparrow \partial_{*} A$ belong to $L^{1}\left(A, \mathbb{R}^{m} ; \mathcal{L}^{m}\right)$ and $L^{1}\left(\partial_{*} A, \mathbb{R}^{m} ; \mathcal{H}^{m-1}\right)$, respectively, and $\operatorname{div} v(x)$ is defined for almost all $x \in A$. Moreover, if $\operatorname{div} v$ belongs to $L^{1}\left(A ; \mathcal{L}^{m}\right)$, then

$$
\int_{A} \operatorname{div} v d \mathcal{L}^{m}=\int_{\partial_{*} A} v \cdot \nu_{A} d \mathcal{H}^{m-1} .
$$

The distinguishing feature of both Theorem 1.1 and the Main Theorem is that only the integrability of $\operatorname{div} v$ implies the Gauss-Green formula; the separate integrability of the relevant partial derivatives of $v$ is not required.

We postpone the proof of the Main Theorem to Section 3 below. Observe that the Main Theorem is reduced to Theorem 1.1 when the collection $\left\{K_{i}\right\}$ is empty.

Remark 2.4. Let $E \subset \mathbb{R}^{m}$ and let $n \geq 1$ be an integer. An $x \in E$ is called a singular point of $f: E \rightarrow \mathbb{R}^{n}$ if $f$ is unbounded in each neighborhood of $x$. Note that no isolated point of $E$ is singular.

Under the assumptions of the Main Theorem, $v$ may or may not have singular points in $K_{i}$. However, the unboundedness of $v$ around $K_{i}$ often takes a more definitive form:

(*) For each $x \in K_{i}$ and for each neighborhood $U$ of $x$, the map $v$ is unbounded in $\left(U-K_{i}\right) \cap \mathrm{cl}_{*} A$.

We claim that if $(*)$ is satisfied for each $K_{i}$, then the collection $\left\{K_{i}\right\}$ is finite.

We prove the claim by contradiction. Select $x_{i} \in K_{i}$ and assuming $\left\{x_{i}\right\}$ is an infinite sequence, find a subsequence, still denoted by $\left\{x_{i}\right\}$, that converges to $x \in \operatorname{cl}_{r} A$. By assumption (7) of the Main Theorem, each $K_{i}$ has a neighborhood $U_{i}$ such that $v$ is locally bounded in $\left(U_{i}-K_{i}\right) \cap \mathrm{cl}_{*} A$. It follows from $(*)$ that $U_{i} \cap K_{j}=\emptyset$ for every $j \neq i$; in particular $x \notin \bigcup_{i} K_{i}$. Inductively, we can find disjoint open sets $V_{i}$ so that $K_{i} \subset V_{i} \subset U_{i}$ for $i=1,2, \ldots$ There is a $y_{i} \in\left(V_{i}-K_{i}\right) \cap \mathrm{cl}_{*} A$ so that $\left|x_{i}-y_{i}\right|<1 / i$ and $\left|v\left(y_{i}\right)\right|>i$. Now $V:=\bigcup_{i}\left(V_{i}-\left\{x, y_{i}\right\}\right)$ is an open set containing $\bigcup_{i} K_{i}$ and $v$ is not bounded in $\operatorname{cl}_{*} A-V$. This contradicts assumption (6) of the Main Theorem. 
A similar argument shows that [12, Theorem 5.4] and [10, Gauss-Green Theorem] apply only to vector fields with finitely many singular points - an observation which simplifies dramatically the statements and proofs of these results.

Example 2.5. Let $A:=B(\mathbf{0}, 1)$, and define $v: \mathrm{cl}_{*} A \rightarrow \mathbb{R}^{m}$ by the formula

$$
v(x):= \begin{cases}x|x|^{-m} & \text { if } x \neq \mathbf{0} \\ 0 & \text { if } x=\mathbf{0} .\end{cases}
$$

A direct calculation shows that $\operatorname{div} v(x)=0$ for each $x \in A-\{\mathbf{0}\}$, and that $\int_{\partial_{*} A} v \cdot \nu_{A} d \mathcal{H}^{m-1}=\|A\|$. Clearly, (7) is the only assumption of the Main Theorem which is not satisfied.

The next example shows that identity (2.1) may hold for vector fields that do not satisfy assumptions of the Main Theorem.

Example 2.6. Let $m=2$ and $p>0$. For $x=(\xi, \eta)$ in $\mathbb{R}^{2}$, let

$$
v(x)= \begin{cases}|x|^{-p}(\eta,-\xi) & \text { if } x \neq \mathbf{0}, \\ 0 & \text { if } x=\mathbf{0} .\end{cases}
$$

A direct calculation shows that $\operatorname{div} v(x)=0$ for each $x \in \mathbb{R}^{2}-\{\mathbf{0}\}$, and

$$
\int_{\partial_{*} B(\mathbf{0}, r)} v \cdot \nu_{B(\mathbf{0}, r)} d \mathcal{H}^{2}=0
$$

for each $r>0$. It follows from Theorem 1.1 that identity (2.1) holds for each $A \in \mathcal{B} \mathcal{V}$ whose topological boundary does not contain $\mathbf{0}$. Yet, $v$ does not satisfy condition (7) of the Main Theorem when $p \geq 2$.

Note that for $A:=B(\mathbf{0}, 1)$ and $1 \leq p<2$, the Main Theorem applies to the vector field $v$ of Example 2.6, although the coordinates of $v$ are not BV functions.

Remark 2.7. A simple modification of the proof presented in Section 3 below shows that the Main Theorem remains valid when conditions (4) and (7) are replaced, respectively, by conditions:

$\left(4^{*}\right)$ each $K_{i}$ is nonempty and $\partial_{*} A$ does not accumulate about $K_{i}$;

$\left(7^{*}\right)$ there are decreasing functions $\beta_{i}: \mathbb{R}_{+} \rightarrow \mathbb{R}_{+}$such that $\int_{0}^{1} \beta_{i}(r) d r<\infty$ and if $y$ approaches $K_{i}, i=1,2, \ldots$, then

or

$$
|v(y)|=o(1) \beta_{i}\left[d\left(y, K_{i}\right)\right] d\left(y, K_{i}\right)^{t+2-m}
$$

$$
|v(y)|=O(1) \beta_{i}\left[d\left(y, K_{i}\right)\right] d\left(y, K_{i}\right)^{t+2-m}
$$

according to whether $\mathcal{M}^{* t}\left(K_{i}\right)>0$ or $\mathcal{M}^{* t}\left(K_{i}\right)=0$, respectively.

Only the obvious reformulations of Lemmas 3.1, 3.2, and 3.4 below must be proved. We leave the details to the reader.

Example 2.8. Let $A$ be the bounded BV set of Example 1.9, and let

$$
v(x):= \begin{cases}x|x|^{-3 / 2} & \text { if } x \in \mathbb{R}^{2}-\{\mathbf{0}\} \\ 0 & \text { if } x=\mathbf{0} .\end{cases}
$$

A direct calculation shows that $\operatorname{div} v$ belongs to $L^{1}\left(A ; \mathcal{L}^{2}\right)$ while $v$ does not belong to $L^{1}\left(\partial_{*} A, \mathbb{R}^{2} ; \mathcal{H}^{1}\right)$. Since condition $\left(7^{*}\right)$ holds with $\beta(r):=r^{-3 / 4}$, it is easy to 
see that condition $\left(4^{*}\right)$ is the only unsatisfied assumption of the Main Theorem modified according to Remark 2.7 .

\section{Proof of the Main Theorem}

Lemma 3.1. Let $E \subset \mathbb{R}^{m}$ be measurable, and let $K \subset \mathbb{R}^{m}$ be a compact set with $\mathcal{M}^{* t}(K)<\infty$ for $0 \leq t<m-1$. Assume $v: E \rightarrow \mathbb{R}^{m}$ is measurable, and

$$
|v(y)|=O(1) d(y, K)^{t+1-m} \quad \text { as } \quad y \rightarrow K .
$$

Then $\lim \int_{B(K, r)}|v| d \mathcal{L}^{m}=0$ as $r>0$ approaches zero.

Proof. Choose an $r>0$ so that $|B(K, s)| / s^{m-t}<c<\infty$ whenever $0<s \leq r$. Making $r$ smaller and $c$ larger, we obtain

$$
\begin{aligned}
\int_{B(K, r)}|v(y)| d y & \leq c \sum_{i=0}^{\infty} \int_{B\left(K, r 2^{-i}\right)-B\left(K, r 2^{-i-1}\right)} d(y, K)^{t+1-m} d y \\
& \leq c \sum_{i=0}^{\infty}\left(r 2^{-i-1}\right)^{t+1-m}\left|B\left(K, r 2^{-i}\right)\right| \\
& \leq 2^{m-1-t} c \sum_{i=0}^{\infty}\left(r 2^{-i}\right) \frac{\left|B\left(K, r 2^{-i}\right)\right|}{\left(r 2^{-i}\right)^{m-t}}<\left(2^{m-t} c^{2}\right) r
\end{aligned}
$$

Lemma 3.2. Let $A \in \mathcal{B} \mathcal{V}$, and let $K \subset \mathbb{R}^{m}$ be a compact set with $\mathcal{M}^{* t}(K)<\infty$ for $0 \leq t<m-1$. Assume the map $v: \mathrm{cl}_{*} A \rightarrow \mathbb{R}^{m}$ is $\mathcal{H}^{m-1}$ measurable, and

$$
|v(y)|=O(1) d(y, K)^{t+1-m} \quad \text { as } \quad y \rightarrow K .
$$

If $K$ is transverse to $\partial_{*} A$, then

$$
\lim _{r \rightarrow 0+} \int_{B(K, r) \cap \partial_{*} A}|v| d \mathcal{H}^{m-1}=0 .
$$

Proof. By the definition of transversality and our assumptions, there are $c>0$ and a decreasing function $\beta: \mathbb{R}_{+} \rightarrow \mathbb{R}_{+}$with $\int_{0}^{1} \beta(r) d r<\infty$ such that given a sufficiently small positive number $r$,

$$
\mathcal{H}^{m-1}\left[B(K, s) \cap \partial_{*} A\right] \leq c \beta(s) s^{m-t} \quad \text { and } \quad|v(y)| \leq c d(y, K)^{t+1-m}
$$

for each positive $s \leq r$ and each $y \in B(K, r)$. Select such an $r>0$, and let

$$
B_{i}:=B\left(K, r 2^{-i}\right)-B\left(K, r 2^{-i-1}\right)
$$

for $i=0,1, \ldots$ As $B(K, r)=K \cup \bigcup_{i=0}^{\infty} B_{i}$ and $\mathcal{H}^{m-1}(K)=0$, we obtain

$$
\begin{aligned}
\int_{B(K, r) \cap \partial_{*} A}|v| d \mathcal{H}^{m-1} & \leq c \sum_{i=0}^{\infty} \int_{B_{i} \cap \partial_{*} A} d(y, K)^{t+1-m} d \mathcal{H}^{m-1}(y) \\
& \leq c^{2} \sum_{i=0}^{\infty}\left(r 2^{-i-1}\right)^{t+1-m} \beta\left(r 2^{-i}\right)\left(r 2^{-i}\right)^{m-t} \\
& \leq 2^{m} c^{2} \sum_{i=0}^{\infty} \int_{r 2^{-i-1}}^{r 2^{-i}} \beta(s) d s=2^{m} c^{2} \int_{0}^{r} \beta(s) d s
\end{aligned}
$$


Lemma 3.3. Let $0 \leq t \leq m-1$, and let $K$ be a compact set. Then $B(K, r) \in \mathcal{B} \mathcal{V}$ for $\mathcal{L}^{1}$ almost all $r>0$, and

$$
\liminf _{r \rightarrow 0+} \frac{\|B(K, r)\|}{r^{m-1-t}} \leq(m-t) \mathcal{M}^{* t}(K) .
$$

Proof. Since $x \mapsto d(x, K)$ is a Lipschitz function whose Lipschitz constant is one, the coarea theorem [3, Section 5.5] implies that $B(K, r) \in \mathcal{B} \mathcal{V}$ for $\mathcal{L}^{1}$ almost all $r>0$, and that

$$
P(r):=\int_{0}^{r}\|B(K, s)\| d s \leq|B(K, r)|
$$

for all $r>0$. Integrating by parts yields

$$
\begin{aligned}
\int_{\delta}^{r} & \|B(K, s)\| s^{t+1-m} d s \\
& =P(r) r^{t+1-m}-P(\delta) \delta^{t+1-m}-(t+1-m) \int_{\delta}^{r} P(s) s^{t-m} d s \\
& \leq r \frac{|B(K, r)|}{r^{m-t}}+(m-1-t) \int_{\delta}^{r} \frac{|B(K, s)|}{s^{m-t}} d s
\end{aligned}
$$

for all $r>\delta>0$. As there is nothing to prove otherwise, assume $\mathcal{M}^{* t}(K)<\infty$. Given $\varepsilon>0$, find an $R>0$ so that

$$
\frac{|B(K, s)|}{s^{m-t}}<\mathcal{M}^{* t}(K)+\varepsilon
$$

for each positive $s<R$, and observe that for $0<\delta<r<R$,

$$
\int_{\delta}^{r} \frac{|B(K, s)|}{s^{m-t}} d s \leq r\left[\mathcal{M}^{* t}(K)+\varepsilon\right] .
$$

Letting $\delta \rightarrow 0$, we obtain

$$
\limsup _{r \rightarrow 0+} \frac{1}{r} \int_{0}^{r} \frac{\|B(K, s)\|}{s^{m-1-t}} d s \leq(m-t)\left[\mathcal{N}^{* t}(K)+\varepsilon\right],
$$

and by the arbitrariness of $\varepsilon$,

$$
\liminf _{r \rightarrow 0+} \frac{\|B(K, r)\|}{r^{m-1-t}} \leq \limsup _{r \rightarrow 0+} \frac{1}{r} \int_{0}^{r} \frac{\|B(K, s)\|}{s^{m-1-t}} d s \leq(m-t) \mathcal{M}^{* t}(K) .
$$

Lemma 3.4. Let $A \in \mathcal{B V}$, and let $K \subset \mathbb{R}^{m}$ be a compact set with $\mathcal{M}^{* t}(K)<\infty$ for $0 \leq t<m-1$. Assume the map $v: \operatorname{cl}_{*} A \rightarrow \mathbb{R}^{m}$ is $\mathcal{H}^{m-1}$ measurable, and that either of the following conditions is satisfied:

(i) $|v(y)|=o(1) d(y, K)^{t+1-m}$ as $y \rightarrow K$.

(ii) $\mathcal{M}^{* t}(K)=0$ and $|v(y)|=O(1) d(y, K)^{t+1-m}$ as $y \rightarrow K$.

If $K$ is transverse to $\partial_{*} A$, then there is a sequence $\left\{r_{i}\right\}$ in $\mathbb{R}_{+}$such that

$$
\lim \left\|A \cap B\left(K, r_{i}\right)\right\|=0 \quad \text { and } \quad \lim \int_{\partial_{*}\left[A \cap B\left(K, r_{i}\right)\right]}|v| d \mathcal{H}^{m-1}=0 .
$$


Proof. By Lemma 3.3, there is a decreasing sequence $\left\{r_{i}\right\}$ in $\mathbb{R}_{+}$such that

$$
\lim r_{i}=0 \quad \text { and } \quad \lim \frac{\left\|B\left(K, r_{i}\right)\right\|}{r_{i}^{m-1-t}} \leq m \mathcal{M}^{* t}(K) ;
$$

in particular, $\lim \left\|A \cap B\left(K, r_{i}\right)\right\|=0$ by [13, Proposition 1.9.2]. Choose $\varepsilon>0$ and let $b:=1+m \mathcal{M}^{* t}(K)$. If condition (i) holds find an integer $k \geq 1$ so that

$$
|v(y)| \leq \frac{\varepsilon}{b} d(y, K)^{t+1-m} \text { and }\left\|B\left(K, r_{i}\right)\right\| \leq b r_{i}^{m-1-t}
$$

whenever $d(y, K)<r_{k}$ and $i \geq k$. For $i \geq k$,

$$
\begin{aligned}
\int_{\partial_{*} B\left(K, r_{i}\right) \cap c l_{*} A}|v| d \mathcal{H}^{m-1} & \leq \frac{\varepsilon}{b} \int_{\partial_{*} B\left(K, r_{i}\right)} d(y, K)^{t+1-m} d \mathcal{H}^{m-1}(y) \\
& =\frac{\varepsilon}{b} r_{i}^{t+1-m}\left\|B\left(K, r_{i}\right)\right\| \leq \varepsilon .
\end{aligned}
$$

If condition (ii) holds, there are $c>0$ and integer $k \geq 1$ such that

$$
|v(y)| \leq c d(y, K)^{t+1-m} \text { and }\left\|B\left(K, r_{i}\right)\right\| \leq \frac{\varepsilon}{c} r_{i}^{m-1-t}
$$

whenever $d(y, K)<r_{k}$ and $i \geq k$. For $i \geq k$ we obtain as before

$$
\int_{\partial_{*} B\left(K, r_{i}\right) \cap c l_{*} A}|v| d \mathcal{H}^{m-1} \leq \varepsilon
$$

and by the arbitrariness of $\varepsilon$,

$$
\lim \int_{\partial_{*} B\left(K, r_{i}\right) \cap c l_{*} A}|v| d \mathcal{H}^{m-1}=0 .
$$

The lemma follows from Lemma 3.2 and the inclusion

$$
\partial_{*}\left[A \cap B\left(K, r_{i}\right)\right] \subset\left[\mathrm{cl}_{*} A \cap \partial_{*} B\left(K, r_{i}\right)\right] \cup\left[B\left(K, r_{i}\right) \cap \partial_{*} A\right],
$$

established in [13, Proposition 1.8.5].

Lemma 3.5. If $A_{1}, A_{2}, \ldots$ are $B V$ sets and $\sum_{i=1}^{\infty}\left\|A_{i}\right\|<\infty$, then $A=\bigcup_{i=1}^{\infty} A_{i}$ is a $B V$ set with $\|A\| \leq \sum_{i=1}^{\infty}\left\|A_{i}\right\|$, and the set $\partial_{*} A-\bigcup_{i=1}^{\infty} \partial_{*} A_{i}$ is $\mathcal{H}^{m-1}$ negligible.

Proof. As all is clear if $m=1$, we assume $m \geq 2$. By the lower semicontinuity of perimeter [3, Section 5.2.1],

$$
\|A\| \leq \liminf _{k}\left\|\bigcup_{i=1}^{k} A_{i}\right\| \leq \liminf _{k} \sum_{i=1}^{k}\left\|A_{i}\right\|=\sum_{i=1}^{\infty}\left\|A_{i}\right\|<\infty,
$$

and the isoperimetric inequality [3, Section 5.6.2] implies $|A|<\infty$. Thus $A$ is a BV set, and a direct verification shows

$$
\partial_{*} A \subset\left(\bigcup_{i=1}^{k} \partial_{*} A_{i}\right) \cup \partial_{*} \bigcup_{i=k+1}^{\infty} A_{i} \subset\left(\bigcup_{i=1}^{\infty} \partial_{*} A_{i}\right) \cup \partial_{*} \bigcup_{i=k+1}^{\infty} A_{i} .
$$

Hence $\partial_{*} A-\bigcup_{i=1}^{\infty} \partial_{*} A_{i} \subset \partial_{*} \bigcup_{i=k+1}^{\infty} A_{i}$ for $k=1,2, \ldots$ The lemma follows:

$$
\underset{k}{\limsup } \mathcal{H}^{m-1}\left(\partial_{*} \bigcup_{i=k+1}^{\infty} A_{i}\right)=\limsup \left\|\bigcup_{i=k+1}^{\infty} A_{i}\right\| \leq \lim _{k} \sum_{i=k+1}^{\infty}\left\|A_{i}\right\|=0 .
$$


Proof of the Main Theorem. As $v$ is continuous $\mathcal{H}^{m-1}$ almost everywhere in $\mathrm{cl}_{*} A$, it is both $\mathcal{L}^{m}$ and $\mathcal{H}^{m-1}$ measurable. Conditions (6) and (7) of the Main Theorem and Lemma 3.1 imply $v \in L^{1}\left(A, \mathbb{R}^{m} ; \mathcal{L}^{m}\right)$. Choose positive numbers $\varepsilon$ and $\delta$, and using Lemma 3.4, find $r_{i}>0$ so that

$$
\left\|A \cap B\left(K_{i}, r_{i}\right)\right\|<\delta 2^{-i} \text { and } \int_{\partial_{*}\left[A \cap B\left(K_{i}, r_{i}\right)\right]}|v| d \mathcal{H}^{m-1}<\varepsilon 2^{-i} .
$$

By Lemma 3.5, the set $A_{\delta}:=A \cap \bigcup_{i} B\left(K_{i}, r_{i}\right)$ is a BV set with $\left\|A_{\delta}\right\| \leq \delta$, and

$$
\int_{\partial_{*} A_{\delta}}|v| d \mathcal{H}^{m-1} \leq \sum_{i} \int_{\partial_{*}\left[A \cap B\left(K_{i}, r_{i}\right)\right]}|v| d \mathcal{H}^{m-1}<\varepsilon
$$

As $\int_{\partial_{*}\left(A-A_{\delta}\right)}|v| d \mathcal{H}^{m-1}<\infty$ follows from condition (6) of the Main Theorem,

$$
\int_{\partial_{*} A}|v| d \mathcal{H}^{m-1} \leq \int_{\partial_{*} A_{\delta}}|v| d \mathcal{H}^{m-1}+\int_{\partial_{*}\left(A-A_{\delta}\right)}|v| d \mathcal{H}^{m-1}<\infty .
$$

Now assume that $\operatorname{div} v$ belongs to $L^{1}\left(A ; \mathcal{L}^{m}\right)$. For sufficiently small $\delta$, the isoperimetric inequality and absolute continuity of the Lebesgue integral yield

$$
\int_{A_{\delta}}|\operatorname{div} v| d \mathcal{L}^{m}<\varepsilon
$$

Applying Theorem 1.1 to $v\left\lceil\mathrm{cl}_{*}\left(A-A_{\delta}\right)\right.$, we obtain

$$
\begin{aligned}
\left|\int_{\partial_{*} A} v \cdot \nu_{A} d \mathcal{H}^{m-1}-\int_{A} \operatorname{div} v d \mathcal{L}^{m}\right| & =\left|\int_{\partial_{*} A_{\delta}} v \cdot \nu_{A_{\delta}} d \mathcal{H}^{m-1}-\int_{A_{\delta}} \operatorname{div} v d \mathcal{L}^{m}\right| \\
& \leq \int_{\partial_{*} A_{\delta}}|v| d \mathcal{H}^{m-1}+\int_{A_{\delta}}|\operatorname{div} v| d \mathcal{L}^{m}<2 \varepsilon
\end{aligned}
$$

and the Main Theorem follows from the arbitrariness of $\varepsilon$.

\section{Applichtions}

Theorem 4.1 (Integration by parts). Let $U \subset \mathbb{R}^{m}$ be an open set, let $v: U \rightarrow \mathbb{R}^{m}$, and let $E_{c}$ and $E_{d}$ be subsets of $U$ such that

(1) $E_{c}$ is $\mathcal{H}^{m-1}$ negligible, and $E_{d}$ is $\mathcal{H}^{m-1} \sigma$-finite,

(2) $|v(y)-v(x)|=o(1)$ as $y$ approaches $x \in U-E_{c}$,

(3) $|v(y)-v(x)|=O(1)|y-x|$ as $y$ approaches $x \in U-E_{d}$.

Further let $\left\{C_{i}\right\}$ be a countable collection of disjoint subsets of $E_{c}$ satisfying the following conditions:

(4) each $C_{i}$ is nonempty and relatively closed in $U$,

(5) given $C_{i}$ and a compact set $K \subset C_{i}$, there is a $t_{K}$ with $0 \leq t_{K}<m-1$ and $\mathcal{M}^{* t_{K}}(K)<\infty$,

(6) $v$ is locally bounded in $U-V$ for each open set $V$ containing $\bigcup_{i} C_{i}$,

(7) given $C_{i}$ and a compact set $K \subset C_{i}$, as y approaches $K$,

$$
|v(y)|=o(1) d(y, K)^{t_{K}+1-m} \quad \text { or } \quad|v(y)|=O(1) d(y, K)^{t_{K}+1-m}
$$

according to whether $\mathcal{M}^{* t_{K}}(K)>0$ or $\mathcal{M}^{* t_{K}}(K)=0$, respectively.

Then $v \in L_{\text {loc }}^{1}\left(U, \mathbb{R}^{m} ; \mathcal{L}^{m}\right)$. Moreover, if $\varphi: U \rightarrow \mathbb{R}$ is locally bounded and

(i) $|\varphi(y)-\varphi(x)|=o(1)$ as $y$ approaches $x \in U-E_{c}$,

(ii) $|\varphi(y)-\varphi(x)|=O(1)|y-x|$ as $y$ approaches $x \in U-E_{d}$, 
then

$$
\int_{U} \varphi \operatorname{div} v d \mathcal{L}^{m}=-\int_{U} \nabla \varphi \cdot v d \mathcal{L}^{m}
$$

whenever $\operatorname{div} v \in L_{\mathrm{loc}}^{1}\left(U ; \mathcal{L}^{m}\right), \nabla \varphi \in L_{\mathrm{loc}}^{1}\left(U, \mathbb{R}^{m} ; \mathcal{L}^{m}\right)$, and $\varphi v$ has compact support.

Proof. The local integrability of $v$ follows easily from assumptions (1)-(7) and Lemma 3.1. Find an $A \in \mathcal{B} \mathcal{V}$ so that $S:=\operatorname{supp}(\varphi v)$ is contained in int $A$ and $\operatorname{cl} A \subset U$. Since $\varphi$ is bounded on $\operatorname{cl} A$ and $S \cap \partial_{*} A=\emptyset$, it is easy to verify that $w:=(\varphi v) \uparrow \mathrm{cl}_{*} A$, the sets $S \cap E_{c}$ and $S \cap E_{d}$, and the collection $\left\{S \cap C_{i}\right\}$ satisfy conditions (1)-(7) of the Main Theorem. As

$$
\operatorname{div} w=\nabla \varphi \cdot v+\varphi \operatorname{div} v
$$

we see that $\operatorname{div} w \in L^{1}\left(A ; \mathcal{L}^{m}\right)$. Applying the Main Theorem to $w$ and observing that $w\left\lceil\partial_{*} A=0\right.$ completes the argument.

Employing the ideas of [2, Section 4], we apply the integration by parts theorem to removable sets for holomorphic and harmonic functions. The following theorem generalizes the classical results of Besicovitch [1].

Theorem 4.2. Let $U$ be an open subset of the complex plane $\mathbb{C}$, let $f: U \rightarrow \mathbb{C}$, and let $E_{c}$ and $E_{d}$ be subsets of $U$ such that

(1) $E_{c}$ is $\mathcal{H}^{1}$ negligible, and $E_{d}$ is $\mathcal{H}^{1} \sigma$-finite,

(2) $|f(y)-f(x)|=o(1)$ as $y$ approaches $x \in U-E_{c}$,

(3) $|f(y)-f(x)|=O(1)|y-x|$ as $y$ approaches $x \in U-E_{d}$.

Further let $\left\{C_{i}\right\}$ be a countable collection of disjoint subsets of $E_{c}$ satisfying the following conditions:

(4) each $C_{i}$ is nonempty and relatively closed in $U$,

(5) given $C_{i}$ and a compact set $K \subset C_{i}$, there is a $t_{K}$ with $0 \leq t_{K}<1$ and $\mathcal{M}^{* t_{K}}(K)<\infty$,

(6) $f$ is locally bounded in $U-V$ for each open set $V$ containing $\bigcup_{i} C_{i}$,

(7) given $C_{i}$ and a compact set $K \subset C_{i}$, as y approaches $K$,

$$
|f(y)|=o(1) d(y, K)^{t_{K}-1} \quad \text { or } \quad|f(y)|=O(1) d(y, K)^{t_{K}-1}
$$

according to whether $\mathcal{M}^{* t_{K}}(K)>0$ or $\mathcal{M}^{* t_{K}}(K)=0$, respectively.

If the complex derivative $f^{\prime}(z)$ exists for almost all $z \in U$, then $f$ can be redefined on $E_{c}$ so that it is holomorphic in $U$.

Proof. Denote by $g$ and $h$ the real and imaginary part of $f$, respectively. The vector fields $v:=(g,-h), w:=(h, g)$, together with any function $\varphi \in C_{c}^{\infty}(U)$, satisfy the assumptions of Theorem 4.1 ; in particular, $v$ and $w$ belong to $L_{\text {loc }}^{1}\left(U, \mathbb{R}^{2} ; \mathcal{L}^{2}\right)$. Since $\operatorname{div} v=\operatorname{div} w=0$ almost everywhere in $U$ by the Cauchy-Riemann equations, integrating by parts yields

$$
\int_{U} v \cdot \nabla \varphi=-\int_{U} \varphi \operatorname{div} v=0 \text { and } \int_{U} w \cdot \nabla \varphi=-\int_{U} \varphi \operatorname{div} w=0 .
$$

In other words, the pair $(f, g)$ is a distributional solution of the Cauchy-Riemann equations, which form an elliptic system. The regularity theorem [15, Example 8.14] implies that almost everywhere $f$ equals a holomorphic function. As $f$ is continuous outside an $\mathcal{H}^{1}$ negligible set, the theorem is established. 
Theorem 4.3. Let $U \subset \mathbb{R}^{m}$ be an open set, let $u: U \rightarrow \mathbb{R}$ be locally bounded, and let $E_{c}$ and $E_{d}$ be subsets of $U$ such that

(1) $E_{c}$ is $\mathcal{H}^{m-1}$ negligible and relatively closed in $U$, and $E_{d}$ is $\mathcal{H}^{m-1} \sigma$-finite,

(2) $u \in C^{1}\left(U-E_{c}\right)$,

(3) $|\nabla u(y)-\nabla u(x)|=O(1)|y-x|$ as $y$ approaches $x \in U-E_{d}$.

Further let $\left\{C_{i}\right\}$ be a countable collection of disjoint subsets of $E_{c}$ satisfying the following conditions:

(4) each $C_{i}$ is nonempty and relatively closed in $U$,

(5) given $C_{i}$ and a compact set $K \subset C_{i}$, there is a $t_{K}$ with $0 \leq t_{K}<m-1$ and $\mathcal{M}^{* t_{K}}(K)<\infty$

(6) $\nabla u$ is locally bounded in $U-V$ for each open set $V$ containing $\bigcup_{i} C_{i}$,

(7) given $C_{i}$ and a compact set $K \subset C_{i}$, as y approaches $K$,

$|\nabla u(y)|=o(1) d(y, K)^{t_{K}+1-m} \quad$ or $\quad|\nabla u(y)|=O(1) d(y, K)^{t_{K}+1-m}$

according to whether $\mathcal{M}^{* t_{K}}(K)>0$ or $\mathcal{M}^{* t_{K}}(K)=0$, respectively.

If $\triangle u(x)=0$ for almost all $x \in U$, then $u$ can be redefined on $E_{c}$ so that it is harmonic in $U$.

Proof. Choose a $\psi \in C_{c}^{\infty}(U)$, and observe that $v:=\nabla \psi$ and $\varphi:=u$, together with the sets $E_{c}$ and $E_{d}:=E_{c}$, satisfy the assumptions of Theorem 4.1 where the collection $\left\{C_{i}\right\}$ is empty. As $U-E_{c}$ is an open set, $\nabla u$ extended to $U$ by zero satisfies conditions $(2),(3),(6)$, and (7) of Theorem 4.1 with respect to $E_{c}, E_{d}$ and $\left\{C_{i}\right\}$. Thus the extended $\nabla u$ and $\psi$, together with $E_{c}, E_{d}$ and $\left\{C_{i}\right\}$, satisfy all assumptions of Theorem 4.1. Integrating by parts twice yields

$$
\begin{aligned}
\int_{U} u \triangle \psi & =\int_{U} u \operatorname{div}(\nabla \psi)=-\int_{U} \nabla u \cdot \nabla \psi \\
& =-\int_{U} \psi \operatorname{div}(\nabla u)=\int_{U} \psi \triangle u=0,
\end{aligned}
$$

which means that $u$ is the distributional solution of the Laplace equation $\Delta u=0$. Since the Laplace equation is elliptic, the theorem follows from [15, Corollary of Theorem 8.12].

\section{REFERENCES}

[1] A.S. Besicovitch, On sufficient conditions for a function to be analytic, and behaviour of analytic functions in the neighbourhood of non-isolated singular points, Proc. London Math. Soc. 32 (1931), 1-9.

[2] T. De Pauw and W.F. Pfeffer, The Gauss-Green theorem and removable sets for PDEs in divergence form, Adv. Math. 183 (2004), 155-182. MR2038549 (2004k:35035)

[3] L.C. Evans and R.F. Gariepy, Measure Theory and Fine Properties of Functions, CRC Press, Boca Raton, 1992. MR1158660 (93f:28001)

[4] K.J. Falconer, The Geometry of Fractal Sets, Cambridge Univ. Press, Cambridge, 1985. MR0867284 (88d:28001)

[5] H. Federer, Geometric Measure Theory, Springer-Verlag, New York, 1969. MR0257325 (41:1976)

[6] Real flat chains, cochains and variational problems, Indiana Univ. Math. J. 24 (1974), 351-407. MR0348598 (50:1095)

[7] R. Harvey and J.C. Polking, Removable singularities of solutions of linear partial differential equations, Acta Math. 125 (1970), 39-56. MR0279461 (43:5183)

[8] M.W. Hirsch, Differential Topology, Springer-Verlag, New York, 1976. MR0448362 (56:6669)

[9] P. Mattila, Geometry of Sets and Measures in Euclidean Spaces, Cambridge Univ. Press, Cambridge, 1995. MR1333890 (96h:28006) 
[10] D.J.F. Nonnenmacher, Sets of finite perimeter and the Gauss-Green theorem, J. London Math. Soc. 52 (1995), 335-344. MR1356146 (96i:26014)

[11] W.F. Pfeffer, The Gauss-Green theorem in the context of Lebesgue integration, Bull. London Math. Soc. 37 (2005), 81-94. MR2105823 (2005j:26007)

[12] The divergence theorem, Trans. Amer. Math. Soc. 295 (1986), 665-685. MR0833702 (87f:26015)

[13] _ Derivation and Integration, Cambridge Univ. Press, New York, 2001. MR1816996 (2001m:26018)

[14] W. Rudin, Real and Complex Analysis, McGraw-Hill, New York, 1987. MR0924157 (88k:00002)

[15] _ Functional Analysis, McGraw-Hill, New York, 1991. MR1157815 (92k:46001)

Département de mathématiques, Université Catholique de Louvain, 2 Chemin du CyClotron, B-1348 Louvain-LA-Neuve, Belgium

E-mail address: depauw@math.ucl.ac.be

Department of Mathematics, University of California, Davis, California 95616

E-mail address: wfpfeffer@ucdavis.edu; washek@mcn.org 